\title{
Influence of radiative damping on the optical-frequency susceptibility
}

\author{
P. W. Milonni \\ Theoretical Division (T-DOT), Los Alamos National Laboratory, \\ Los Alamos, New Mexico 87545 \\ Robert W. Boyd \\ Institute of Optics, University of Rochester, Rochester, New York 14627 \\ (Date textdate; Received textdate; Revised textdate; Accepted textdate; \\ Published textdate)
}

\begin{abstract}
Motivated by recent discussions concerning the manner in which damping appears in the electric polarizability, we show that (a) there is a dependence of the nonresonant contribution on the damping and that (b) the damping enters according to the "opposite sign prescription." We also discuss the related question of how the damping rates in the polarizability are related to energy-level decay rates.
\end{abstract}




\section{INTRODUCTION}

Several recent papers address the question of how material damping effects should be included in the response of an atom or molecule to an applied electric field [1]-[ $[$ ]. We will consider the simplest case, that of the linear atomic polarizability, which in the absence of damping is given by the Kramers-Heisenberg formula,

$$
\alpha_{i}(\omega)=\frac{e^{2}}{3 \hbar} \sum_{j}\left|\mathbf{r}_{j i}\right|^{2}\left(\frac{1}{\omega_{j i}-\omega}+\frac{1}{\omega_{j i}+\omega}\right)
$$

for state $i$. Here $\omega_{j i}$ and $\mathbf{r}_{j i}$ are the transition (angular) frequency and coordinate matrix element, respectively, between states $i$ and $j$, and the field frequency $\omega$ is assumed to be far removed from any of the atomic transition frequencies $\omega_{j i}$. More generally one associates damping rates $\gamma_{j i}$ with the different transitions and writes

$$
\alpha_{i}(\omega)=\frac{e^{2}}{3 \hbar} \sum_{j}\left|\mathbf{r}_{j i}\right|^{2}\left(\frac{1}{\omega_{j i}-\omega-i \gamma_{j i}}+\frac{1}{\omega_{j i}+\omega+i \xi \gamma_{j i}}\right)
$$

where $\xi=+1$ according to the so-called "opposite sign" prescription and $\xi=-1$ in the "constant sign" prescription. The difference appears only in the nonresonant terms, and is therefore unimportant in most situations. However, the question of which prescription is the correct one raises some interesting points, as we shall see, and the purpose of this paper is to address some of these points as well as to answer the question of whether one should take $\xi=+1$ or $\xi=-1$ in equation (2).

One might ask first whether a damping term should appear at all in the nonresonant part of the Kramers-Heisenberg formula, i.e., whether we should in fact take $\xi=0$ instead of either $\xi=+1$ or $\xi=-1$. An analysis involving the diagonalization of the (two-level) atom-field Hamiltonian in the rotating-wave approximation, for instance, shows that there is no damping term in the nonresonant denominator [6], a result that is certainly accurate for most practical purposes. In a broader context the issue here is an old one. Thus the imaginary part of the polarizability (2) implies an absorption coefficient having the usual Lorentzian form

$$
\frac{\gamma}{\left(\omega-\omega_{0}\right)^{2}+\gamma^{2}}
$$


as well as a nonresonant part

$$
\frac{\gamma}{\left(\omega+\omega_{0}\right)^{2}+\gamma^{2}}
$$

for a transition of frequency $\omega_{0}$ and linewidth $\gamma$, and one might question whether, as a matter of principle, (4) contributes to the absorption lineshape. In his consideration of possible corrections to the Weisskopf-Wigner lineshape, Lamb 7] noted that "such a contribution [as (4)] appears in some derivations," but added that it would be negligible compared with the resonant contribution (3).

The effect of damping on the nonresonant part of the polarizability is not an entirely trivial matter, and the literature relating to the subject reveals significant disagreement on some rather basic aspects of dissipation theory. The purpose of this paper is to address the principal points where there is disagreement and to obtain what we regard as the correct form of the polarizability when damping is included.

In the following section we consider the problem of the electric-dipole interaction of a two-level atom with the quantized electromagnetic field, assuming that all but one of the field modes are initially unoccupied. Using the rotating-wave approximation (RWA) for the atomic source field but not for the applied field, we obtain exactly the result cited earlier [6], and in particular we find that there is no contribution from (radiative) damping to the nonresonant term in the polarizability. In Section III] we go beyond the RWA in the atomic source field and find that the damping now appears in the nonresonant term, and that it does so in accordance with the "opposite sign" prescription. Section IV presents a discussion of these results, including their connection to the classical theory of radiative damping. Section $\square$ focuses on the form of the damping rate $\gamma_{j i}$, and we argue that, contrary to what sometimes appears in the literature, $\gamma_{j i}$ depends on the sum rather than the difference of the level decay rates. Our conclusions are summarized in Section VI. 


\section{DERIVATION OF LINEAR POLARIZABILITY: RWA}

The model we consider is described by the Hamiltonian

$H=\hbar \omega_{0} \sigma_{z}+\sum_{k} \hbar \omega_{k} a_{k}^{\dagger} a_{k}-d E \sigma_{x}=\hbar \omega_{0} \sigma_{z}+\sum_{k} \hbar \omega_{k} a_{k}^{\dagger} a_{k}-i \hbar \sum_{k} C_{k}\left(a_{k}-a_{k}^{\dagger}\right)\left(\sigma+\sigma^{\dagger}\right)$

for a two-level atom (TLA) with transition frequency $\omega_{0}$ and dipole moment $d$ interacting with the electromagnetic field. The $\sigma$ operators are the usual Pauli operators, with $\sigma$ and $\sigma^{\dagger}$ the lowering and raising operators for the TLA. $a_{k}$ and $a_{k}^{\dagger}$ are the annihilation and creation operators for field mode $k$, and $C_{k}=\left(\mathbf{d} \cdot \mathbf{e}_{\mathbf{k}, \lambda} / \hbar\right)\left(2 \pi \hbar \omega_{k} / V\right)^{1 / 2}$, with $V$ the quantization volume. The subscript $k$ denotes $(\mathbf{k}, \lambda)$, where $\mathbf{k}$ is the wave vector assocated with a plane-wave mode of frequency $\omega_{k}=|\mathbf{k}| c$ and $\mathbf{e}_{\mathbf{k}, \lambda}$ is a corresponding polarization unit vector $\left(\mathbf{k} \cdot \mathbf{e}_{\mathbf{k}, \lambda}=0, \mathbf{e}_{\mathbf{k}, \lambda} \cdot \mathbf{e}_{\mathbf{k}, \lambda^{\prime}}^{*}=\delta_{\lambda \lambda^{\prime}}, \lambda=1,2\right)$.

The commutation relations for the atom and field operators give the Heisenberg equations of motion

$$
\begin{gathered}
\dot{\sigma}=-i \omega_{0} \sigma+\sum_{k} C_{k}\left(\sigma_{z} a_{k}-a_{k}^{\dagger} \sigma_{z}\right) \\
\dot{a}_{k}=-i \omega_{k} a_{k}+C_{k}\left(\sigma+\sigma^{\dagger}\right)
\end{gathered}
$$

We have chosen a normal ordering for the field annihilation and creation operators, which is especially useful in the case that the applied field is described by a coherent state [Eq. (11)]. As we are interested only in determining the linear response, the equation of motion for $\sigma_{z}$ will not be needed for our purposes.

The formal solution of equation (17) is

$$
a_{k}(t)=a_{k}(0) e^{-i \omega_{k} t}+C_{k} \int_{0}^{t} d t^{\prime}\left[\sigma\left(t^{\prime}\right)+\sigma^{\dagger}\left(t^{\prime}\right)\right] e^{i \omega_{k}\left(t^{\prime}-t\right)}
$$

In one version of the rotating-wave approximation (RWA) we ignore the coupling between the creation operator for the field and the raising operator for the atom; this corresponds, for the purpose of obtaining the equation of motion for the field operators, to the neglect of the terms $a_{k}^{\dagger} \sigma^{\dagger}$ and $\sigma a_{k}$ in the Hamiltonian (5). In this 
approximation the equation of motion for $\sigma$ becomes

$$
\begin{aligned}
\dot{\sigma}(t)= & -i \omega_{0} \sigma(t)+\sum_{k} C_{k}\left[\sigma_{z}(t) a_{k}(0) e^{-i \omega_{k} t}-a_{k}^{\dagger}(0) \sigma_{z}(t) e^{i \omega_{k} t}\right] \\
& +\sum_{k} C_{k}^{2} \int_{0}^{t} d t^{\prime} \sigma_{z}(t) \sigma\left(t^{\prime}\right) e^{i \omega_{k}\left(t^{\prime}-t\right)}
\end{aligned}
$$

Note that we are not making an RWA in the free-field operators $a_{k}(0)$ and $a_{k}^{\dagger}(0)$, so that both annihilation and creation free-field operators $\left[a_{k}(0)\right.$ and $\left.a_{k}^{\dagger}(0)\right]$ appear in (91).

Taking expectation values over the initial atom-field state on both sides of (9), we have

$$
\begin{aligned}
\langle\dot{\sigma}(t)\rangle= & -i \omega_{0}\langle\sigma(t)\rangle+\sum_{k} C_{k}\left[\left\langle\sigma_{z}(t) a_{k}(0)\right\rangle e^{-i \omega_{k} t}-\left\langle a_{k}^{\dagger}(0) \sigma_{z}(t)\right\rangle e^{i \omega_{k} t}\right] \\
& +\sum_{k} C_{k}^{2} \int_{0}^{t} d t^{\prime}\left\langle\sigma_{z}(t) \sigma\left(t^{\prime}\right)\right\rangle e^{i \omega_{k}\left(t^{\prime}-t\right)}
\end{aligned}
$$

We assume that the initial field state $\left|\psi_{F}\right\rangle$ corresponds to a single occupied mode described by a coherent state with

$$
a_{k}(0)\left|\psi_{F}\right\rangle=\alpha\left|\psi_{F}\right\rangle, \quad\left\langle\psi_{F}\right| a_{k}^{\dagger}(0)=\alpha^{*}\left\langle\psi_{F}\right|
$$

corresponding to the expectation value

$$
\langle E(t)\rangle=i\left(\frac{2 \pi \hbar \omega}{V}\right)^{1 / 2}\left[\alpha e^{-i \omega t}-\alpha^{*} e^{i \omega t}\right] \equiv \mathcal{E}_{0} \cos \omega t
$$

of the applied electric field. Thus

$$
\sum_{k} C_{k}\left[\left\langle\sigma_{z}(t) a_{k}(0)\right\rangle e^{-i \omega_{k} t}-\left\langle a_{k}^{\dagger}(0) \sigma_{z}(t)\right\rangle e^{i \omega_{k} t}\right]=-\frac{i}{\hbar} d_{x} \mathcal{E}_{0} \cos \omega t\left\langle\sigma_{z}(t)\right\rangle
$$

where $d_{x}$ is the component of the dipole matrix element along the direction of the applied field; $d_{x}^{2}=d^{2} / 3$ for the spherically symmetric atom.

We assume that the operator $\sigma_{z}$, which corresponds to the population inversion, changes sufficiently slowly that we may take

$$
\left\langle\sigma_{z}(t) \sigma\left(t^{\prime}\right)\right\rangle \cong\left\langle\sigma_{z}\left(t^{\prime}\right) \sigma\left(t^{\prime}\right)\right\rangle=-\left\langle\sigma\left(t^{\prime}\right)\right\rangle
$$


in the integral appearing in (10). Since we want to obtain the polarizability for the TLA in a particular state, we assume further that the atom remains with high probability in its initial state. Assuming this initial state to be the lower state, we approximate $\left\langle\sigma_{z}(t)\right\rangle$ by -1 , so that, using (13) and the approximation (14), we replace (10) by

$$
\langle\dot{\sigma}(t)\rangle=-i \omega_{0}\langle\sigma(t)\rangle+i \frac{d}{\hbar} d_{x} \mathcal{E}_{0} \cos \omega t-\sum_{k} C_{k}^{2} \int_{0}^{t} d t^{\prime}\left\langle\sigma\left(t^{\prime}\right)\right\rangle e^{i \omega_{k}\left(t^{\prime}-t\right)}
$$

We seek a solution of (15) of the form

$$
\langle\sigma(t)\rangle=s e^{-i \omega t}+r e^{i \omega t}
$$

with $s$ and $r$ constants to be determined. This implies

$$
\begin{aligned}
i\left(\omega_{0}-\omega\right) s e^{-i \omega t}+i\left(\omega_{0}+\omega\right) r e^{i \omega t}= & \frac{i d_{x}}{2 \hbar} \mathcal{E}_{0}\left(e^{-i \omega t}+e^{i \omega t}\right)-\left[\gamma_{-}(\omega)-i \Delta_{-}(\omega)\right] s e^{-i \omega t} \\
& -\left[\gamma_{+}(\omega)-i \Delta_{+}(\omega)\right] r e^{i \omega t}
\end{aligned}
$$

where

$$
\begin{aligned}
\gamma_{ \pm}(\omega) & =\mathcal{R} e \sum_{k} C_{k}^{2} \int_{0}^{t} d t^{\prime} e^{i\left(\omega_{k} \pm \omega\right)\left(t^{\prime}-t\right)} \rightarrow \frac{V}{8 \pi^{3}} \frac{2 \pi}{\hbar V} \int d^{3} k \omega_{k} \sum_{\lambda}\left|\mathbf{d} \cdot \mathbf{e}_{\mathbf{k}, \lambda}\right|^{2} \pi \delta\left(\omega_{k} \pm \omega\right) \\
& =\frac{d^{2}}{4 \pi^{2} \hbar c^{3}} \int d \Omega \Omega^{3} \pi \delta(\Omega \pm \omega) \int_{0}^{\pi} d \theta \sin ^{3} \theta \\
& =\frac{2 d^{2}}{3 \hbar c^{3}} \int_{0}^{\infty} d \Omega \Omega^{3} \delta(\Omega \pm \omega)=\frac{2 d^{2} \omega^{3}}{3 \hbar c^{3}} U( \pm \omega)
\end{aligned}
$$

and

$$
\Delta_{ \pm}(\omega)=\frac{2 d^{2}}{3 \pi \hbar c^{3}} \mathrm{P} \int_{0}^{\infty} \frac{d \Omega \Omega^{3}}{\Omega \pm \omega}
$$

for $t>>1 / \omega$, where $U$ is the unit step function. Note that the damping rate $\gamma_{-}(\omega)$ is frequency-dependent [8]. $\left(\Delta_{ \pm}(\omega)\right.$ is obviously divergent but, as discussed in Section 4, this has no direct bearing on our conclusions regarding the effect of damping on the polarizability.) To obtain the polarizability $\alpha(\omega)$ we write

$$
p=d_{x}\left\langle\sigma_{x}\right\rangle=d_{x}\left(\langle\sigma\rangle+\left\langle\sigma^{\dagger}\right\rangle\right)=2 d_{x} \mathcal{R e}\left[\left(r+s^{*}\right) e^{-i \omega t}\right] \equiv \mathcal{R e}\left[\alpha(\omega) \mathcal{E}_{0} e^{-i \omega t}\right]
$$


for the induced dipole moment. This yields

$$
\alpha(\omega)=\frac{d^{2}}{3 \hbar}\left(\frac{1}{\omega_{0}-\omega-\Delta_{-}(\omega)-i \gamma_{-}(\omega)}+\frac{1}{\omega_{0}+\omega-\Delta_{+}(\omega)+i \gamma_{+}(\omega)}\right)
$$

Note that $\gamma_{+}(\omega)=0$, and that therefore there is no damping contribution to the second (nonresonant) term. $\gamma_{-}\left(\omega_{0}\right)$ is half the radiative decay rate of the upper state in the absence of any applied field.

\section{DERIVATION OF LINEAR POLARIZABILITY WITHOUT RWA}

Let us now recalculate the polarizability, this time retaining both terms inside the integral of equation (8), i.e., without making the RWA in the (source) field produced by the atom under consideration. Then (91) is replaced by

$$
\begin{aligned}
\langle\dot{\sigma}(t)\rangle= & -i \omega_{0}\langle\sigma(t)\rangle-i \frac{d_{x}}{\hbar} \mathcal{E}_{0} \cos \omega t\left\langle\sigma_{z}(t)\right\rangle \\
& +\sum_{k} C_{k}^{2} \int_{0}^{t} d t^{\prime}\left[\left\langle\sigma_{z}(t) \sigma\left(t^{\prime}\right)\right\rangle+\left\langle\sigma_{z}(t) \sigma^{\dagger}\left(t^{\prime}\right)\right\rangle\right] e^{i \omega_{k}\left(t^{\prime}-t\right)} \\
& -\sum_{k} C_{k}^{2} \int_{0}^{t} d t^{\prime}\left[\left\langle\sigma^{\dagger}\left(t^{\prime}\right) \sigma_{z}(t)\right\rangle+\left\langle\sigma\left(t^{\prime}\right) \sigma_{z}(t)\right\rangle\right] e^{-i \omega_{k}\left(t^{\prime}-t\right)}
\end{aligned}
$$

when we take expectation values as before. The approximations tantamount to (14) are

$$
\begin{aligned}
\left\langle\sigma_{z}(t) \sigma\left(t^{\prime}\right)\right\rangle & \cong\left\langle\sigma_{z}\left(t^{\prime}\right) \sigma\left(t^{\prime}\right)\right\rangle=-\left\langle\sigma\left(t^{\prime}\right)\right\rangle \\
\left\langle\sigma_{z}(t) \sigma^{\dagger}\left(t^{\prime}\right)\right\rangle & \cong\left\langle\sigma_{z}\left(t^{\prime}\right) \sigma^{\dagger}\left(t^{\prime}\right)\right\rangle=\left\langle\sigma^{\dagger}\left(t^{\prime}\right)\right\rangle \\
\left\langle\sigma^{\dagger}\left(t^{\prime}\right) \sigma_{z}(t)\right\rangle & \cong\left\langle\sigma^{\dagger}\left(t^{\prime}\right) \sigma_{z}\left(t^{\prime}\right)\right\rangle=-\left\langle\sigma^{\dagger}\left(t^{\prime}\right)\right\rangle \\
\left\langle\sigma\left(t^{\prime}\right) \sigma_{z}(t)\right\rangle & \cong\left\langle\sigma\left(t^{\prime}\right) \sigma_{z}\left(t^{\prime}\right)\right\rangle=\left\langle\sigma\left(t^{\prime}\right)\right\rangle
\end{aligned}
$$

where we use the equal-time identities $\sigma_{z}(t) \sigma(t)=-\sigma(t) \sigma_{z}(t)=-\sigma(t)$. Using these approximations in (22), together with the approximation $\left\langle\sigma_{z}(t)\right\rangle \cong-1$ in the second term, we obtain the non-RWA extension of (15):

$$
\begin{aligned}
\langle\dot{\sigma}(t)\rangle= & -i \omega_{0}\langle\sigma(t)\rangle+\frac{i d_{x}}{\hbar} \mathcal{E}_{0} \cos \omega t+\sum_{k} C_{k}^{2} \int_{0}^{t} d t^{\prime}\left[-\left\langle\sigma\left(t^{\prime}\right)\right\rangle+\left\langle\sigma^{\dagger}\left(t^{\prime}\right)\right\rangle\right] e^{i \omega_{k}\left(t^{\prime}-t\right)} \\
& -\sum_{k} C_{k}^{2} \int_{0}^{t} d t^{\prime}\left[-\left\langle\sigma^{\dagger}\left(t^{\prime}\right)\right\rangle+\left\langle\sigma\left(t^{\prime}\right)\right\rangle\right] e^{-i \omega_{k}\left(t^{\prime}-t\right)}
\end{aligned}
$$


It is important to note that in equations (23) we have used the commutation relations between $\sigma_{z}(t)$ and $\sigma(t), \sigma^{\dagger}(t)$, and have obviously not made the approximation that $\sigma_{z}$ could be replaced by -1 . The latter approximation is made only in the second term of (22), where $\sigma_{z}$ multiplies the applied field but no atom operator, so that the approximation does not violate the commutation relations from which we obtained the equations of motion. The two approximations are different: that made in (23) assumes that $\sigma_{z}(t)$ varies little on time scales $\sim 1 / \omega_{k}$ for field frequencies $\omega_{k} \sim \omega$ that will contribute significantly to the variation of $\langle\sigma(t)\rangle$, whereas that made in replacing $\left\langle\sigma_{z}(t) \mathcal{E}_{0} \cos \omega t\right.$ by $-\mathcal{E}_{0} \cos \omega t$ assumes that the atom remains with high probability in its lower state because the field frequency lies outside the absorption linewidth. The difference between these two approximations involving $\sigma_{z}$ turns out to be irrelevant for the final results when the RWA is made, as is clear from (14).

We again have a solution of the form (16), now with $s$ and $r$ satisfying

$$
\begin{aligned}
X s+U r^{*} & =\frac{d_{x}}{2 \hbar} \mathcal{E}_{0} \\
V s+Y r^{*} & =\frac{d_{x}}{2 \hbar} \mathcal{E}_{0}
\end{aligned}
$$

where

$$
\begin{aligned}
X & =\omega_{0}-\omega-\left[\Delta_{-}(\omega)-\Delta_{+}(\omega)\right]-i\left[\gamma_{-}(\omega)+\gamma_{+}(\omega)\right] \\
U & =\left[\Delta_{-}(\omega)-\Delta_{+}(\omega)\right]+i\left[\gamma_{-}(\omega)+\gamma_{+}(\omega)\right] \\
Y & =\omega_{0}+\omega+\left[\Delta_{-}(\omega)-\Delta_{+}(\omega)\right]+i\left[\gamma_{-}(\omega)+\gamma_{+}(\omega)\right] \\
V & =\left[\Delta_{-}(\omega)-\Delta_{+}(\omega)\right]+i\left[\gamma_{-}(\omega)+\gamma_{+}(\omega)\right]
\end{aligned}
$$

Assuming that $\gamma_{ \pm}(\omega)$ and $\Delta_{ \pm}(\omega)$ are small in magnitude compared to $\omega_{0} \pm \omega$, we have

$$
\begin{aligned}
& s \cong \frac{d_{x} \mathcal{E}_{0}}{2 \hbar} \frac{1}{X}=\frac{d_{x} \mathcal{E}_{0}}{2 \hbar} \frac{1}{\omega_{0}-\omega-\left[\Delta_{-}(\omega)-\Delta_{+}(\omega)\right]-i\left[\gamma_{-}(\omega)+\gamma_{+}(\omega)\right]} \\
& r^{*} \cong \frac{d_{x} \mathcal{E}_{0}}{2 \hbar} \frac{1}{Y}=\frac{d_{x} \mathcal{E}_{0}}{2 \hbar} \frac{1}{\omega_{0}+\omega+\left[\Delta_{-}(\omega)-\Delta_{+}(\omega)\right]+i\left[\gamma_{-}(\omega)+\gamma_{+}(\omega)\right]}
\end{aligned}
$$


and, from (201),

$$
\begin{aligned}
\alpha(\omega)= & \frac{d^{2} / 3 \hbar}{\omega_{0}-\omega-\left[\Delta_{-}(\omega)-\Delta_{+}(\omega)\right]-i\left[\gamma_{-}(\omega)+\gamma_{+}(\omega)\right]} \\
& +\frac{d^{2} / 3 \hbar}{\omega_{0}+\omega+\left[\Delta_{-}(\omega)-\Delta_{+}(\omega)\right]+i\left[\gamma_{-}(\omega)+\gamma_{+}(\omega)\right]}
\end{aligned}
$$

\section{DISCUSSION}

In contrast to the RWA result (21),$\Delta_{ \pm}(\omega)$ and $\gamma_{ \pm}(\omega)$ appear in both the resonant and nonresonant terms of (28). Consider first the physical significance of $\Delta_{ \pm}(\omega)$, assuming that the frequency $\omega$ of the initially occupied field mode is sufficiently close to $\omega_{0}$ that we may take

$$
\Delta_{-}(\omega) \approx \Delta_{-}\left(\omega_{0}\right)=\frac{2 d^{2}}{3 \pi \hbar c^{3}} \mathrm{P} \int_{0}^{\infty} \frac{d \Omega \Omega^{3}}{\Omega-\omega_{0}}
$$

and focusing only on the resonant term in $\alpha(\omega)$. In a more complete analysis involving the transformation from the fundamental minimal coupling form of the Hamiltonian to the electric dipole form, it is found that the additional term $2 \pi \int d^{3} \mathbf{P}^{\perp} \cdot \mathbf{P}^{\perp}$ appearing in the transformed Hamiltonian has the effect of replacing (29) by [9]

$$
\Delta_{-}\left(\omega_{0}\right) \approx \frac{2 d^{2} \omega_{0}^{2}}{3 \pi \hbar c^{3}} \mathrm{P} \int_{0}^{\infty} \frac{d \Omega \Omega}{\omega-\omega_{0}}
$$

With this modification it is seen that $\Delta\left(\omega_{0}\right) \equiv \Delta_{-}\left(\omega_{0}\right)-\Delta_{+}\left(\omega_{0}\right)$ is simply the (unrenormalized) TLA radiative frequency shift, i.e., the difference in the radiative level shifts of the two levels [10]. In general, however, the approximation (29) is not applicable, and the radiative level shifts $\hbar \Delta_{ \pm}(\omega)$ depend on the frequency of the initially occupied mode. In the polarizability (28) the frequency shift $\Delta(\omega) \equiv \Delta_{-}(\omega)-\Delta_{+}(\omega)$ adds to the field frequency $\omega$ in both the resonant and nonresonant terms, whereas in the RWA $\Delta_{+}(\omega)$ does not appear in the resonant term and $\Delta_{-}(\omega)$ does not appear in the nonresonant term. In other words, the RWA does not correctly include the radiative frequency shift as the difference in the radiative level shifts of the TLA.

The expressions for the level shifts $\hbar \Delta_{ \pm}(\omega)$ are specific to the TLA model, but are easily generalized to the case of a real atom. This extension, even with the 
standard renormalization procedures, still leaves us with divergent level shifts in the nonrelativistic approximation. A high-frequency cutoff $m c^{2} / \hbar$ results in Bethe's approximation to the Lamb shift [10]. Since this procedure is very well known, and we are in any case only concerned with the form in which the radiative corrections appear in the polarizability, and not their numerical values, we will simply assume henceforth that the frequency shift has been accounted for in writing $\omega_{0} \pm \omega$.

Thus

$$
\alpha(\omega)=\frac{d^{2}}{3 \hbar}\left(\frac{1}{\omega_{0}-\omega-i \gamma(\omega)}+\frac{1}{\omega_{0}+\omega+i \gamma(\omega)}\right)
$$

where $\gamma(\omega)=\gamma_{-}(\omega)+\gamma_{+}(\omega)$. Like $\Delta(\omega)$, $i \gamma(\omega)$ is effectively an addition to the applied field frequency $\omega$. Unlike the frequency shift, however, the damping rate $\gamma(\omega)$ is half the sum of the decay rates $\gamma_{ \pm}(\omega)$ of the two levels. Of course the decay rate $\gamma_{+}(\omega)$ of the ground state in our two-level model is zero but, as discussed in the next section, (31) is valid more generally when the decay rate of the lower level of the transition is not zero. That is, the damping rate appearing in the contribution to the polarizability from any given transition involves half the sum of the decay rates of the two levels of the transition.

Regardless of whether the lower-level decay rate vanishes, the non-RWA result (31) shows that both the resonant and nonresonant contributions to the polarizability have a nonvanishing damping term in their denominators, this damping term being half the upper-level decay rate. In particular, it is seen that the damping appears according to the "opposite sign prescription," i.e., $\xi=+1$ is the correct choice in the dispersion formula (2). The same conclusion was reached by different lines of reasoning by Buckingham and Fischer [2].

Note that, if $\gamma$ is taken to be a (positive) constant, independent of frequency, then the opposite sign prescription is consistent with the causality requirement that the polarizability should be analytic in the upper half of the complex $\omega$ plane 11]. But in general the decay rates are in fact frequency-dependent [8], and causality is ensured only if the model used to calculate $\gamma(\omega)$ is itself causal. In fact, as recalled below, radiative damping provides an example in which this is not the case. 
In one approach to a classical calculation of the natural lineshape, one considers the solution $x(t)=A_{0} e^{-\gamma t} \sin \left(\omega_{0} t\right)$ of a damped dipole oscillator with resonance frequency $\omega_{0}$. The lineshape is taken to be proportional to the squared modulus of the Fourier transform

$$
a(\omega)=\frac{A_{0}}{2 \pi} \int_{0}^{\infty} d t e^{-\gamma t} e^{i \omega t} \sin \left(\omega_{0} t\right) \propto\left(\frac{1}{\omega_{0}-\omega-i \gamma}+\frac{1}{\omega_{0}+\omega+i \gamma}\right)
$$

and is seen to be consistent with the "opposite sign" prescription. In contrast to this, an old paper by Weisskopf [12] implies the result

$$
a(\omega) \propto\left(\frac{1}{\omega_{0}-\omega-i \gamma}-\frac{1}{\omega_{0}+\omega-i \gamma}\right)
$$

which is consistent with the "constant sign" prescription. However, since this result is based on the integral appearing in (32), it seems that (33) involves a sign error or perhaps just a typographical error.

Since the absorption coefficient may for our purposes be taken to be proportional to the imaginary part of $\alpha(\omega)$, equation (31) implies an absorption lineshape proportional to

$$
L(\omega)=\frac{\gamma}{\left(\omega_{0}-\omega\right)^{2}+\gamma^{2}}-\frac{\gamma}{\left(\omega_{0}+\omega\right)^{2}+\gamma^{2}}
$$

The same result, for $\gamma$ taken to be a constant, was obtained on the basis of the Lorentz model by Van Vleck and Weisskopf [13], who noted that the minus sign in the nonresonant term "must be used because the excitation of the molecule is here accompanied by emission rather than absorption of a light quantum," a process which is excluded when the RWA is made [14].

It is also of interest to compare the result (31) with the corresponding result given by the classical theory of radiative damping based on the equation

$$
\ddot{x}+\omega_{0}^{2} x-\frac{2 e^{2}}{3 m c^{3}} \dddot{x}=e \mathcal{E}_{0} \cos \omega t
$$

The polarizability of the classical dipole oscillator described by this equation is

$$
\begin{aligned}
\alpha_{c l}(\omega) & =\frac{e^{2} / m}{\omega_{0}^{2}-\omega^{2}-\frac{2}{3}\left(i e^{2} / m c^{3}\right) \omega^{3}} \\
& =\frac{e^{2}}{2 m \omega_{0}^{\prime}}\left(\frac{1}{\omega_{0}^{\prime}-\omega-i \gamma_{c l}(\omega)}+\frac{1}{\omega_{0}^{\prime}+\omega+i \gamma_{c l}(\omega)}\right)
\end{aligned}
$$


where $\omega_{0}^{\prime}=\sqrt{\omega_{0}^{2}-\gamma_{c l}^{2}(\omega)}$ and $\gamma_{c l}(\omega)=\left(e^{2} / 3 m c^{3}\right) \omega^{2}$. The replacements $e^{2} / 2 m \omega_{0}^{\prime} \rightarrow$ $e^{2} f_{1} / 2 m \omega_{0}^{\prime}$ and $e^{2} \omega^{2} / 3 m c^{3} \rightarrow e^{2} f_{2} \omega^{2} / 3 m c^{3}$, where $f_{1}=2 m \omega_{0}^{\prime} d^{2} / e^{2} \hbar$ and $f_{2}(\omega)=$

$\left(2 m \omega d^{2} / e^{2} \hbar\right)$, make the classical result (35) equivalent to (31). These replacements involving effective oscillator strengths $f_{1}$ and $f_{2}$ are the usual substitutions required to put classical oscillator results in agreement with some of the corresponding quantummechanical expressions.

The $\omega^{3}$ in the denominator of (36), or in other words the third derivative of $x$ in equation (35), leads to a pole in the upper half of the complex $\omega$ plane, thus violating the causality requirement that the polarizability be analytic in the upper half-plane. The nonrelativistic theory of radiative reaction is well known to be acausal, but the acausality occurs on such a short time scale that relativistic quantum effects must be taken into account. For most practical purposes the acausality is of no consequence. Thus, for instance, equation (36) leads to the correct extinction coefficient $(\propto \omega \mathcal{I} \mathrm{m}[\alpha(\omega)])$ due to Rayleigh scattering.

\section{RELATION OF DAMPING IN THE POLARIZABILITY TO LEVEL DE- CAY RATES}

These considerations are easily extended beyond the two-level model, with the result that the linear atomic polarizability has the form

$$
\alpha_{i}(\omega)=\frac{1}{3 \hbar} \sum_{j}\left|\mathbf{r}_{j i}\right|^{2}\left(\frac{1}{\omega_{j i}-\omega-i \gamma_{j i}(\omega)}+\frac{1}{\omega_{j i}+\omega+i \gamma_{j i}(\omega)}\right)
$$

The damping rate $\gamma_{j i}(\omega)$ has a "dephasing" contribution associated, for instance, with elastic collisions, as well as a contribution associated with the decay rate of the atomic states $i$ and $j$. Here we consider only the latter contribution, which is due to radiative decay and other loss processes. In the case of radiative decay, for instance, $\gamma_{j i}$ is found, by a straightforward multilevel generalization of the calculations in the preceding sections, to be half the sum of the radiative decay rates associated with the 
two states $i$ and $j$ :

$$
\gamma_{j i}(\omega)=\frac{2 e^{2} \omega^{3}}{3 \hbar c^{3}}\left(\sum_{E_{j}>E_{m}}\left|\mathbf{r}_{j m}\right|^{2}+\sum_{E_{i}>E_{m}}\left|\mathbf{r}_{i m}\right|^{2}\right)
$$

where $E_{m}$ denotes the energy of state $m$. If we replace $\omega^{3}$ by $\omega_{0}^{3}$ in this formula, we obtain half the spontaneous decay rate of state $j$ in the case that the field is initially in the vacuum state. This result was obtained, for example, by Weisskopf and Wigner 15], Landau [16], and many others [9]. The same conclusion is reached in the more general case where the energy levels decay by nonradiative channels: $\gamma_{j i}(\omega)$ is half the sum of the total decay rates of the states $i$ and $j$.

Various authors, however, have calculated or assumed - erroneously, in our opinion-that $\gamma_{j i}$ involves the difference in the decay rates of the states $i$ and $j$ [17], [18], 19]. In addition to the Heisenberg-picture calculation leading to the conclusion that $\gamma_{j i}$ involves the sum rather than the difference of energy-level decay rates, as presented in this paper, the following simple argument can be used. Let $c_{i}(t)$ and $c_{j}(t)$ be the (Schrödinger-picture) probability amplitudes for states $i$ and $j$, and let $\gamma_{i}$ and $\gamma_{j}$ be the decay rates of these states. Then $c_{i}^{*}(t) c_{j}(t)$ and $c_{i}(t) c_{j}^{*}(t)$, which determine the polarizability, decay at $\exp \left[-\frac{1}{2}\left(\gamma_{i}+\gamma_{j}\right) t\right]$, and so the linewidth in the polarizability must involve the sum of $\gamma_{i}$ and $\gamma_{j}$ rather than the difference. Sushchinskii 17], for instance, expresses his results in terms of complex energies $E_{i}^{\prime}=E_{i}-\frac{1}{2} i \Gamma_{i}$ and their differences $E_{i}^{\prime}-E_{j}^{\prime}=E_{i}-E_{j}-\frac{1}{2} i\left(\Gamma_{i}-\Gamma_{j}\right)$, whereas the appropriate differences entering into the polarizability are $E_{i}^{*}-E_{j}^{\prime}$ and $E_{i}^{\prime}-E_{j}^{\prime *}$.

Finally we note that Andrews et al. [19] have stated a polarizability sum rule which in the simplest case of the linear polarizability can be expressed as $\sum_{i} \alpha_{i}(\omega)=0$. A physical plausibility argument for this sum rule can be adduced as follows. If $p_{i}$ is the probability that the atom is in state $i$, then the linear polarizability at field frequency $\omega$ is

$$
\alpha(\omega)=\sum_{i} p_{i} \alpha_{i}(\omega)
$$

Consider the idealized limit in which all the $p_{i}$ are equal. Then the polarizability and therefore the induced emission or absorption rate at frequency $\omega$ becomes proportional 
to just $\sum_{i} \alpha_{i}(\omega)$. But if all the states are equally populated the net induced emission and absorption rate must vanish, implying the polarizability sum rule conjectured by Andrews et al. From the expression (37) it follows that this sum rule is statisfied only if $\gamma_{j i}$ is symmetric in $i$ and $j$, i.e., $\gamma_{j i}$ must involve the sum rather than the difference of $\gamma_{i}$ and $\gamma_{j}$. (We note that, in the case of the constant sign prescription for the damping terms in the polarizability, the polarizability sum rule would be satisfied only if $\gamma_{j i}$ were antisymmetric in $i$ and $j$.)

\section{SUMMARY}

Following a standard, nonrelativisitic approach, we have considered specifically the case of a two-level atom interacting with the quantized electromagnetic field, one mode of which is initially occupied and described by a coherent state. Working in the Heisenberg picture, we calculated the polarizability with and without making the RWA for the atomic source field. In the RWA we obtained a known result, and in particular the nonresonant contribution to the polarizability was found to have no damping factor in its denominator. Going beyond the RWA, however, we found that both the resonant and nonresonant contributions to the polarizability have the radiative damping rate in their denominators, and that the polarizability has a form that is consistent with the so-called opposite sign prescription for including the damping.

The radiative frequency shift appearing in the non-RWA expression for the polarizability depends on the radiative level shifts in the correct way, i.e., it is the difference of the two level shifts. The damping rate appearing in the non-RWA expression for the polarizability is half the sum of the radiative decay rates of the two levels, in contrast to the difference of the decay rates that has been obtained or assumed in some treatments. The fact that the polarizability depends symmetrically on the decay rates of the energy levels is consistent with the polarizability sum rule of Andrews et al. 19] when the (correct) opposite sign prescription is used. 


\section{Acknowledgement}

We thank D. L. Andrews, L. C. Dávila Romero, and G. E. Stedman for helpful correspondence, and P. R. Berman and J. H. Carter for useful discussions and suggestions. R. W. Boyd gratefully acknowledges support by ONR under award N00014-02-1-0797, by DoE under award DE-FG02-01ER15156, and by ARO under award DAAD19-01-1-0623.

[1] D. L. Andrews, S. Naguleswaran, and G. E. Stedman, Phys. Rev. A57, 4925 (1998).

[2] A. D. Buckingham and P. Fischer, Phys. Rev. A61, 035801 (2000).

[3] G. E. Stedman, S. Naguleswaran, D. L. Andrews, and L. C. Dávila Romero, Phys. Rev. A63, 047801 (2001).

[4] A. D. Buckingham and P. Fischer, Phys. Rev. A63, 047802 (2001).

[5] D. L. Andrews, L. C. Dávila Romero, and G. E. Stedman, Phys. Rev. A67, 055801 (2003).

[6] R. Loudon, The Quantum Theory of Light (Clarendon Press, Oxford, 1973), p. 192. See also G. S. Agarwal and R. W. Boyd, Phys. Rev. A67, 043821 (2003), for a different approach to the same result.

[7] W. E. Lamb, Jr., Phys. Rev. 85, 259 (1952).

[8] See G. S. Agarwal and R. W. Boyd, Reference [6].

[9] See, for instance, P. W. Milonni, Phys. Rep. 25, 1 (1976).

[10] See, for instance, P. W. Milonni, The Quantum Vacuum. An Introduction to Quantum Electrodynamics (Academic Press, San Diego, 1994), Section 4.9.

[11] See, for instance, H. M. Nussenzveig, Causality and Dispersion Relations (Academic Press, New York, 1972).

[12] V. Weisskopf, Phys. Z. 34, 1 (1933).

[13] J. H. Van Vleck and V. F. Weisskopf, Rev. Mod. Phys. 17, 227 (1945). 
[14] Van Vleck and Weisskopf went on to show that, when the dipole orientations or phases after each collision are treated statistically according to the Boltzmann distribution, rather than assumed to be random as in the original Lorentz treatment, one obtains an absorption lineshape in which the nonresonant contribution is added rather than subtracted from the resonant term. Then, instead of the vanishing absorption predicted by the original Lorentz treatment when the absorption frequency $\omega_{0} \rightarrow 0$, one obtains the Debye lineshape.

[15] V. F. Weisskopf and E. Wigner, Z. Phys. 63, 54 (1930).

[16] L. D. Landau, Z. Phys. 45, 430 (1927).

[17] M. M. Sushchinskii, Raman Spectra of Molecules and Crystals (Israel Program for Scientific Translations, New York, 1972), p. 38.

[18] L. Hecht and L. D. Barron, Mol. Phys. 79, 887 (1993); Chem. Phys. Lett. 225, 519 (1994).

[19] D. L. Andrews, L. C. Dávila Romero, and G. E. Stedman, Phys. Rev. A67, 055801 (2003). 